\title{
PID Controller Tuning of a Boiler Control System Using Immune Algorithm Typed Neural Network
}

\author{
Dong Hwa Kim \\ Dept. of Instrumentation and Control Eng., Hanbat National University, \\ 16-1 San Duckmyong-Dong Yuseong-Gu, Daejon City, Korea, 305-719. \\ kimdh@hanbat.ac.kr, ial.hanbat.ac.kr \\ Tel: +82-42-821-1170, Fax: +82-821-1164
}

\begin{abstract}
Dead time processes exist widely in many types of systems such as chemical processes, and the main steam temperature control system of the thermal power plant. A PID Controllers have been used to operate these systems. However, it is very difficult to achieve an optimal PID gain with no experience since the gain of the PID controller has to be manually tuned by trial and error. This paper suggests a tuning method of the PID Controller for a process with long dead time using an immune algorithm typed neural network, through computer simulation. Tuning results of immune algorithms based neural network are compared with the results of genetic algorithm.
\end{abstract}

\section{Introduction}

When using a PID controller in these plants, the plant is generally controlled without consideration of disturbance rejection. Therefore, an industrial experience is required for tuning in these systems: [1]. Traditionally, PID controllers applied to these plants are tuned with a reduction of gain so that overall stability can be obtained. This results in poor performance of control. Failure to tune in control will cause an inevitable plant shutdown, and a loss of production and considerable damage to the plant may result. An effective tuning is required to maintain the system reliability and stability following a system disturbance: [1], [2] In this paper auto-tuning scheme of the PID controller using reference model and immune network is suggested and simulated for an effective control of dead time process.

\section{Problems of the PID Controller on the Dead Time Process}

There are many well known PI and PID tuning formulas for stable processes. However, PID tuning formulas for unstable processes, complex plants, and dead time process are less common. Up to this time, many sophisticated tuning algorithms have been tried an attempt to improve the PID controller performance under such difficult conditions since the control performance of the system depends on the P, I, D parameter gains. In the PID tuning methods, the method proposed by Ziegler Nichols (1942) needs the ultimate gain and the period of the ultimate oscillation at stability limit. But it is difficult to determine their exact values experimentally in real 
processes, since the oscillatory operation must be avoided. Hence, a new idea for automatic tuning of the PID control parameters, auto-tuning, is required. In utility boiler of Fig. 1, the boiler-turbine model is given as [1], [2]

\section{Multiobjective Controller Design of Boiler-Turbine System Using Immune Algorithm Based Neural Structure}

\subsection{The Response of Immune System}

Generally the concentration of $\mathrm{i}$-th antibody, which is denoted by $\delta_{i}$, is calculated as follows [7], [8]:

$$
\frac{d S_{i}(t)}{d t}=\left(\alpha \sum_{j=1}^{N} m_{j i} \delta_{j}(t)-\alpha \sum_{k=1}^{N} m_{i k} \delta_{k}(t)+\beta m_{i}-\gamma_{i}\right) \delta_{i}(t), \quad \frac{d \delta_{i}(t)}{d t}=\frac{1}{1+\exp \left(0.5-\frac{d S_{i}(t)}{d t}\right)}
$$

In Eq., $m_{j i}$ denotes affinities between antibody $j$ and antibody $i$ (i.e. the degree of interaction), $m_{i}$ represents affinities between the detected antigens and antibody $i$, respectively. The affinity $m_{\alpha \beta}$ between antibody $\alpha$ and antibody $\beta$ is given as $m_{\alpha \beta}=1 /\{1+\Omega(\alpha \beta)\}, \quad \Omega(\alpha \beta)=f(x)=\left[f_{1}(x)+f_{2}(x)+f_{3}(x)\right]$, where $\Omega_{\alpha \beta} \quad$ is an information which obtained by antibody $\alpha$ and antibody $\beta$. Generally $m_{\alpha \beta}$ is given by range of $0-1$.

\subsection{Controller Design by Immune Algorithm}

In Fig. 1, $r_{=1,2,3}$ is reference input over $0, y_{i=1,2,3}$ is plant output. Immune algorithm typed neural network has the following function in each layer;

Layer 1. As function comparing the reference input with the output of the given plant, comparing result, $r_{=1,2,3}$ is selected for affinity in sub-function and it is defined by the followings.

Layer 2. As layer for computing coupling degree between inputs of multivariable system as Fig. 1, the sub-function defined as $f_{a}, f_{b}, f_{c}$ is used for coupling degree as;

$$
f_{a}=f_{1}\left(x_{1}\right)+f_{2}\left(x_{2}\right), f_{b}=f_{1}\left(x_{1}\right)+f_{3}\left(x_{3}\right), f_{c}=f_{2}\left(x_{2}\right)+f_{3}\left(x_{3}\right) .
$$

Layer 3. As layer for detecting error between reference input and plant output, the following equation is defined as

$$
f_{i}\left(x_{i}\right)=\sum_{t=0}^{n}\left(\left|r_{1}-y_{1}\right|\right) / n, i=1,2,3, \text { if }\left|r_{i}-y_{i}\right|_{t} \geq r_{i} \text { then } f_{i}\left(x_{i}\right)_{t}=1 \text {, else } f_{i}\left(x_{i}\right)_{t}=\left|r_{i}-y_{i}\right|_{t}, i=1,2,3
$$

Layer 4. They provide stimulation action by algorithms defined as

$$
f_{r i} \text { : if } f_{a}<f_{b} \text { then stimulation } f_{i}\left(x_{i}\right) \text {, else stimulation } f_{i}\left(x_{i}\right), i=1,2,3 \text {. }
$$


Layer 5. Affinity is calculated with $H_{S}=f_{1}\left(x_{1}\right)+f_{2}\left(x_{2}\right)+f_{3}\left(x_{3}\right)$. When the value of $H_{S}$ is smaller, more satisfactory results is obtained.

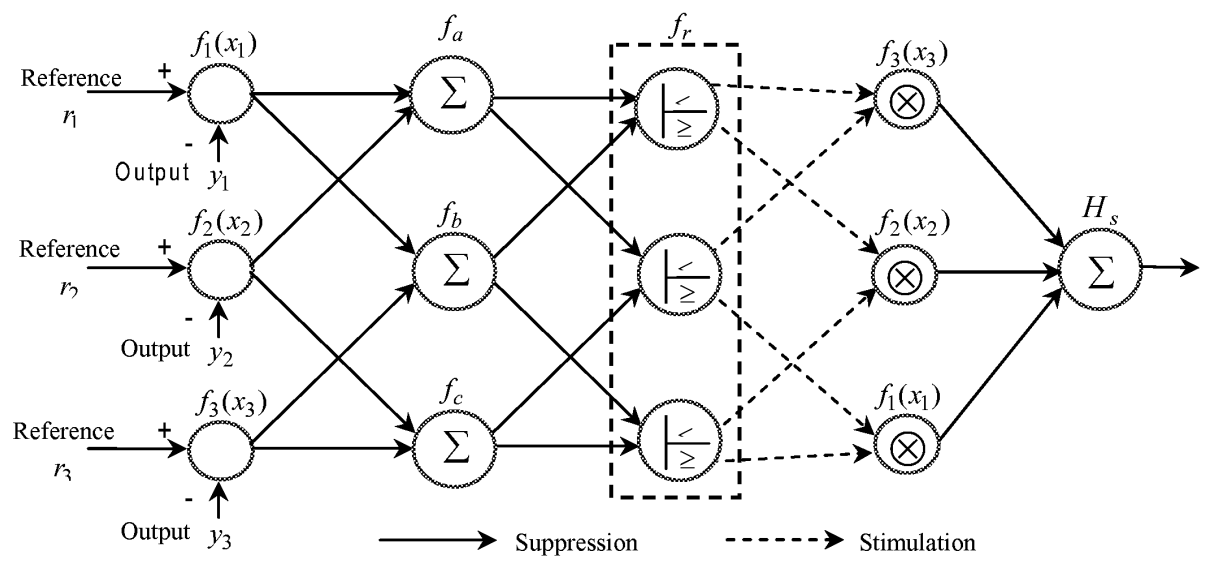

Fig. 1. Flow diagram of controller
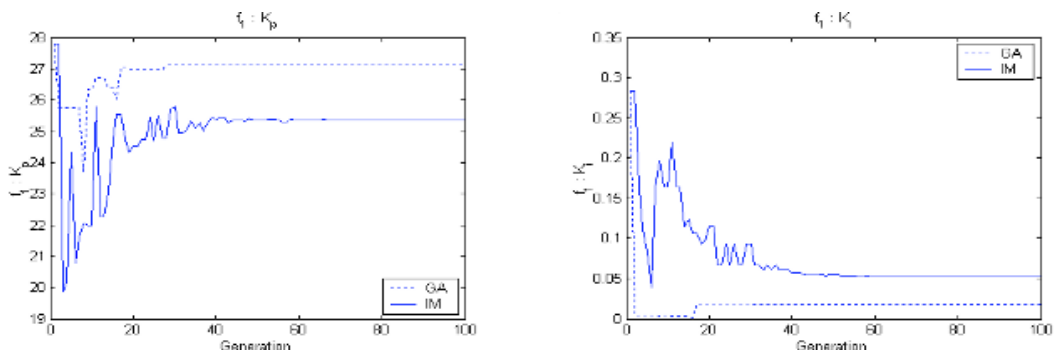

Fig. 2. Graph of sub-function $f_{1}, K_{p}$

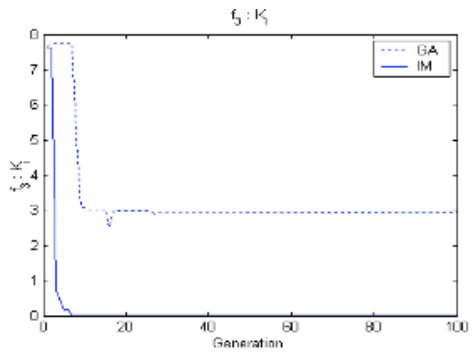

Fig. 4. Graph of sub-function $f_{3}, K_{i}$

Fig. 3. Graph of sub-function $f_{1}, K_{i}$

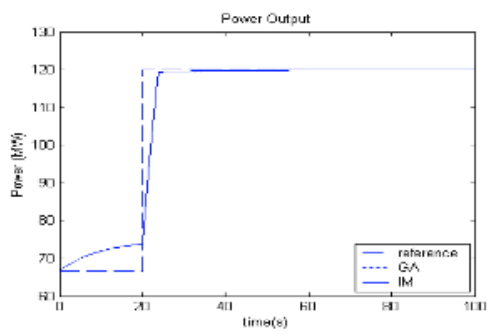

Fig. 5. Pressure response 


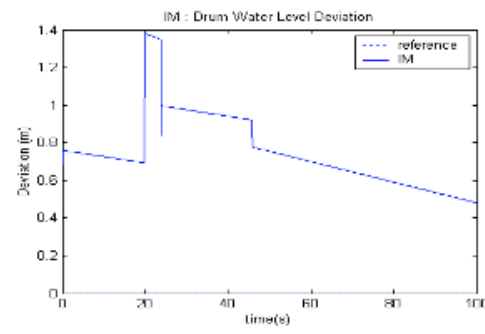

Fig. 6. Water level deviation using immune algorithm

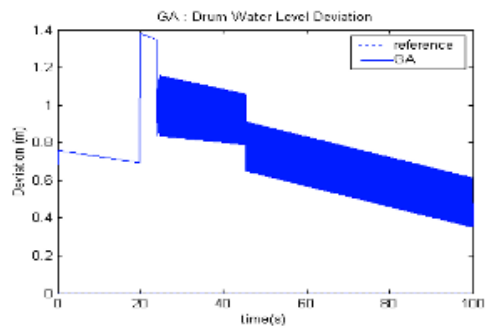

Fig. 7. Water level deviation using genetic algorithm

\section{Simulation and Discussion}

Figs. 4-7 represent the variation results of sub-function, $f_{1}, f_{2}, f_{3}$ and the PI controller parameters, $K_{p}, K_{i}$ is compared on immune algorithm and genetic algorithm. Fig. 5 is pressure responses and Fig. 6 obtained by immune algorithm has no oscillation against many oscillation based on genetic algorithm as shown in Fig. 7. The range of initial value on parameter PI is P: 0-10, I: 0-5.

\section{Conclusion}

The immune system possesses a self organizing and distributed memory. Therefore, it is thus adaptive to its external environment and allows a PDP (parallel distributed processing) network to complete patterns against the environmental situation. This paper suggests a tuning method of the PI Controller for a steam temperature process with long dead time using an immune algorithm typed neural network, through computer simulation. Tuning results by immune algorithms based neural network are compared with the results of genetic algorithm.

\section{References}

1. Teng Fong-Chwee: Self-tuning PID controllers for dead time process. IEEE Trans., vol. 35, no. 1. (1988) 119-125

2. J. D. Farmer, N. H. Packard and A. S. Perelson: The immune system, adaptation, and machine learning, Physica. vol. D, no. 22. (1986) 187 - 204

3. Dong Hwa Kim: Intelligent tuning of a PID controller using a immune algorithm. Trans. KIEE , vo. 51-D, no.1. (2002) 78-91 